\title{
Effect of Growth Promoting Bacteria on the Growth Rate and Lipid Content of Microalgae Chorella sp in Sludge Liquor of Anaerobic Digester of Dairy Manure
}

\author{
Wahyunanto A. Nugroho", Fatma R.Nurlaili", Yusuf Hendrawan", Bambang D. Argo ${ }^{\#}$ \\ ${ }^{\#}$ Department of Agricultural Engineering, Brawijaya University,Jl Veteran 1, Malang, Indonesia. 65145 \\ E-mail:wahyunanto@ub.ac.id
}

\begin{abstract}
Microalgae are microorganisms that have potential as biofuel feedstock since it has relatively high content of lipid. To be economically feasible, microalgae has to be cultivated in lower operational cost. Liquor of anaerobic sludge is one of the choices since it contents high concentration in nutrient. To boost its productivity, growth promoting bacteria (GPB) was added in the medium. The objective of this research is to observe the influence of GPB on the growth rate and oil content of the algae. The experiment used three treatment in respect to the dose of GPB added to the medium, and was done in triplicate. The three treatments are A3 (spiked with 3 $\mathrm{ml}$ of GPB inoculum), A6 (spiked with $6 \mathrm{ml}$ of GPB inoculum) and A9 (spiked with $9 \mathrm{ml}$ of GPB inoculum), and one more treatment as a control (A0). Result showed that the higher the dose of GPB added, the higher the lipid content of microalgae. For the growth rate of microalga, the highest growth rate was observed on A6 treatment, while the lowest one was observed on the control.
\end{abstract}

Keywords - microalgae; growth promoting bacteria; sludge liquor.

\section{INTRODUCTION}

The world energy demand is continuing to increase. Fossil fuel remains the biggest fuel provider for the world. Since it is non-renewable source of energy, it is necessary to observe another source of energy that is renewable. Biofuel is known as a biomass-derived fuel. Biodiesel can be considered as the dominant fuel among all biofuel. Until recently, palm oil is still the largest feed stock for biodiesel. With some environment issue related to palm oil plantation, observation to seek another type of biodiesel feed stock is essential.

Microalgae can be considered as an ideal and potential organism to be used as feed stock for biofuel production. The lipid content in the dry mass of microalgae could reach above $50 \%$. Microalgae can grow rapidly, which is only about ten days to be harvested. Using mathematical estimation, when it is processed into biodiesel oil, its oil productivity could reach for about $120,000 \mathrm{~kg}$ of biodiesel / ha of land per year. It is about 20 times as much as the productivity of palm plantation $(5,800 \mathrm{~kg}$ of biodiesel / ha of land per year) and even compared to castor oil $(1.500 \mathrm{~kg}$ of biodiesel / ha of land per year) [1]. With its potency, as well as its estimated lower production cost, microalgae has been attracting worldwide attention to alter, or at least to complement fossil fuel. Moreover, microalgae contain suitable types of lipid to be converted in esterification process into biodiesel [2].

In order to grow well, it is important to set the growth medium into its optimal growth condition. Besides $\mathrm{pH}$, temperature and light intensity, another essential factor to ensure the microalgae growth is nutrient. So far, the nutrient of microalgae is taken from synthesized fertilizer that makes its production cost is relatively high. To suppress the production cost of microalgae production, an alternative nutrient provider is observed. Wastewaters from food or agricultural industries are among the candidate medium since it contain rich of nutrients $[3,4]$.

Dairy manure is one of the environmental problems when it is not well handled. One of the ways to solve the problem is by converting it microbially into biogas. A biogas digester has effluent in the form of sludge. Normally, the sludge is processed into compost while the liquor is left to flow in the drainage system. In several investigations, this sludge liquor still contains high concentration of nutrients. Rather than let the nutrient leaks into drainage system, utilize the nutrient for microalgae production is a smart way; it safe environment while in the same time we can produce an alternative energy.

In the development, researchers begin to investigate another way to boost to production of microalgae when environmental factors have been optimized. By boosting the 
growth, microalgae is expected to reach a higher productivity in less time. In the research by bahsan, in order to enhance the growth rate, Azospirillum $s p$ was added in the medium. Azospirillum $s p$ is known as a terrestrial microorganism to produce indole acetic acid, which is a growth hormone in plant. Because of its character, Azospirrillum $s p$ is then mentioned as a growth promoting bacterium (GPB). Several past researches papers show that the growth rate of microalgae could be increased when microalgae cultivation was added with GPB $[5,6]$.

The objective of this research is to observe the influence of GPB on the growth rate and oil content of the algae while it is cultivating with the nutrient from sludge liquor of anaerobic digester of dairy manure.

\section{MATERIAL AND METHOD}

\section{A. Material}

Chlorella sp from Indonesian Brackish Water Aquaculture Bureau (Situbondo) and Azospirillum $s p$ as the growth promoting bacterium (GPB) from Laboratory of Soil Microbiology, Brawijaya University, Indonesia. The sludge liquor was taken from an anaerobic digester of dairy manure, which is in one of the village in Malang district.

\section{B. Measurement of Light Intensity, Medium Temperature and $\mathrm{pH}$ of the Medium.}

The light intensity, temperature and $\mathrm{pH}$ of the medium were not definitely controlled. It was necessary to make sure that all those three parameters were under optimum condition, which is 1000lux to 8000 lux for light intensity, $25^{\circ} \mathrm{C}$ to $35^{\circ} \mathrm{C}$ for medium temperature, and 6.5 to 8.0 for the medium $\mathrm{pH}$. Lux meter was used to measure the intensity. The temperature was measured using standard thermometer. The $\mathrm{pH}$ was measured using $\mathrm{pH}$ meter.

\section{Determination of Initial Nutrient Concentration}

Ammonium concentration was determined using Nesslerization spectrophotometric method. The nitrate was measured using colorimetric method using phenol sulfonic acid. The ortho-phosphate concentration was measured using stannous chloride method. All those nutrient concentration was measured every two days.

\section{Measurement of Chlorella sp and Azospirillum sp}

The number of Chlorella sp was counted every day at $04.00 \mathrm{pm}$ using haemocytometer under microscope, while the Azospirillum sp number was counted using the method in every four day.

\section{E. Determination of Lipid Content}

The lipid content was measured using soxhlet method. The biomass that was analyzed for lipid content was taken a day after it reached the peak population.

\section{F. Experimental Set Up}

Cultivation of microalgae was carried out in about $500 \mathrm{ml}$ of medium using 1 litre of glass. The medium was $500 \mathrm{ml}$ of distilled water and spiked with $5 \mathrm{ml}$ of sterilized sludge liquor as the nutrient provider. All medium were added Chlorella sp culture with targeted initial concentration was 1 $\mathrm{x} 106 \mathrm{cell} / \mathrm{ml}$. The concentration of GPB inoculum was $1 \mathrm{x}$ $108 \mathrm{cfu} / \mathrm{ml}$. For the treatment, the samples were added with GPB in various amounts as follows: A0 (control / no GPB added, A3 (spiked with $3 \mathrm{ml}$ of GPB inoculum), A6 (spiked with $6 \mathrm{ml}$ of GPB inoculum) and A9 (spiked with $9 \mathrm{ml}$ of GPB inoculum). All treatments were done in triplicate.

\section{G. Statistical Analysis}

The data was analysed using SPSS 15.0 software. The significance of the growth rate and lipid content was analysed using one-way ANOVA with a significance level of 0.05 and followed by Tukey post-hoc analysis.

\section{RESULT AND DISCUSSION}

\section{A. Condition of Culture Medium}

Fig. 1 shows the average medium temperature of each medium for 10 days. It shows that the medium temperature was on the allowed temperature for microalgae growth. As well as the $\mathrm{pH}$, it was also on the allowed $\mathrm{pH}$ which ranged from 7.00 to 8.0. The light intensity was also in the optimum condition, which was 5000 to 7600 lux. The microalgae received this light intensity for about 16 hours a day with the support of fluorescence lamp. The data for $\mathrm{pH}$ and light intensity is not shown in this article.

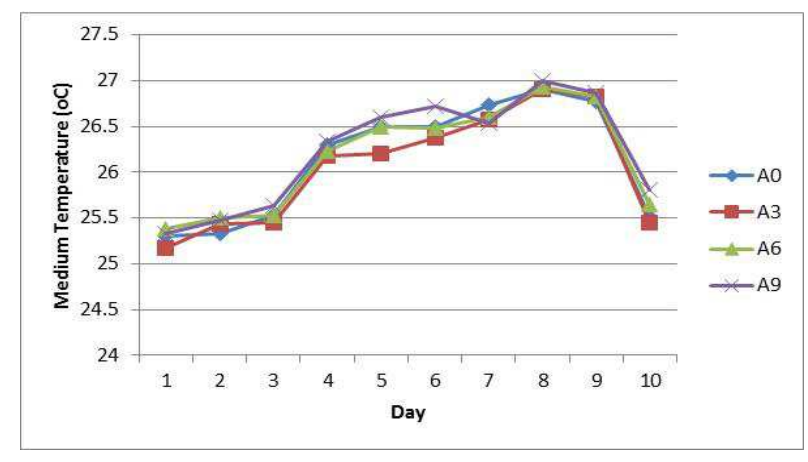

Fig. 1 Average daily temperature of the medium

\section{B. Population of Chlorella sp}

Figure 2 shows population of Chlorella sp during the cultivation. On the first three day, the growth rate was relatively small. This was because Chlorella sp. was requiring adaptation to the new environment. The population of Chlorella sp increased significantly on day-3, and it continued until 6 . In general, the peak population of all treatment occurred in day-8, except for the treatment with 9 $\mathrm{ml}$ of GPB inoculum addition. This treatment was also reached the highest population of microalgae followed by the $6 \mathrm{~mL}$ of GPB inoculum addition, which were $9.24 \times 10^{8} \mathrm{cell} / \mathrm{ml}$ and $9.15 \times 10^{8} \mathrm{cell} / \mathrm{ml}$ respectively. However these two treatments were not significantly different statistically. In other world, treatment with $6 \mathrm{ml}$ and $9 \mathrm{ml}$ of GPB addition had the same peak population number of microalgae. The lowest population in the peak point was the control. 


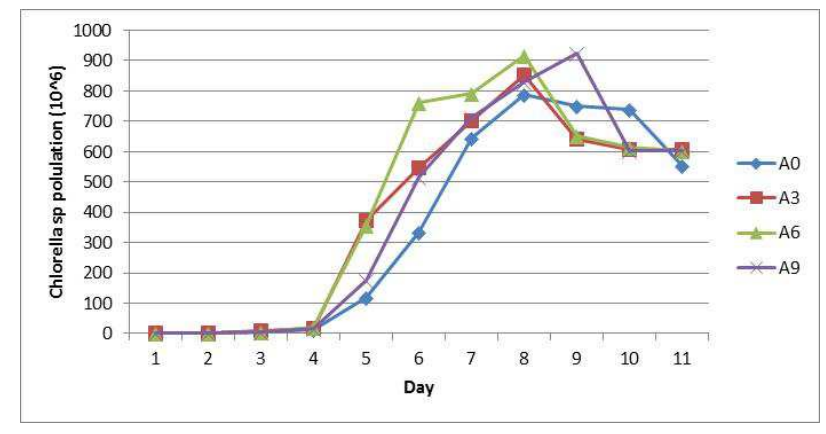

Fig. 2 Population of Chlorella sp

This finding agree with the research of Bashan [5] where the GPB has significantly increase the production of microalgae when they were coupled in the wastewater as a culture medium. In this coupled culture, Azospirillum $s p$ became an agent to support the growth (bio-control agent) and buffer that interacted with microalgae to increase the population of microalgae. According to Bashan, this GPB worked in several mechanisms. Firstly, it is able to decompose organic matter, which might still left in the medium. The decomposition by-products, such as $\mathrm{CO} 2$, ammonium, nitrate, and phosphate, are could become additional nutrients for the microalgae. Since the sludge liquor was not be sterilized, bacterium other than Azospirillum $s p$ could also did this decomposition. This additional nutrient might has extended the growth of microalgae, especially in the last treatment. The second mechanism is by secreting growth hormone, namely IAA (Indole Acetic Acid) that has well known as the hormone to promote growth in terrestrial plant. Since microalgae are an autotrophic organism, this hormone is also applied for microalgae.

After the peak point, the population of Chlorella sp. decreased significantly. This is due to the unavailability of nutrients in the culture media that support the generation of new cells of Chlorella sp. This reason is supported by the finding that after day- 8 the nitrogen concentration decreased (Fig. 7).

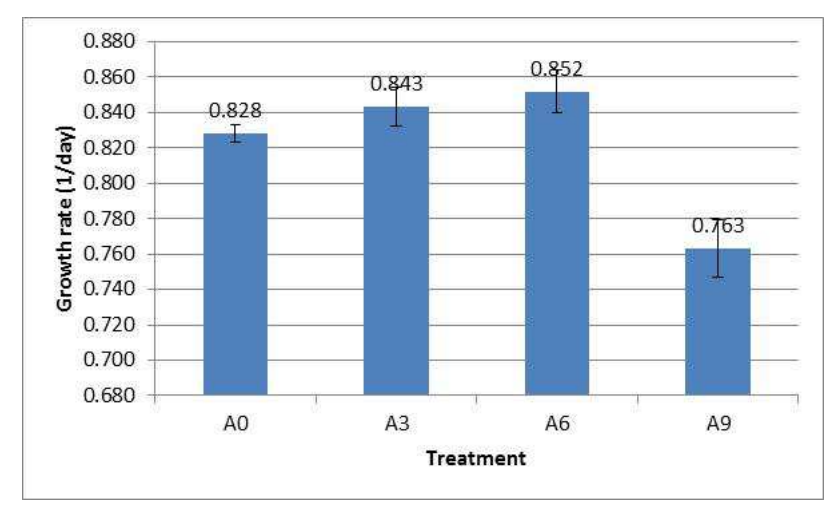

Fig. 3 Average growth rate of microalgae

The average growth rate of each treatment was calculated using the initial population data as the day- 0 and the peak population as the final population. Figure 3 demonstrate the average growth rate of each treatment. The highest growth rate was reached by $6 \mathrm{ml}$ GPB addition $\left(0.852 \mathrm{~d}^{-1}\right)$ followed by $3 \mathrm{ml} \mathrm{GPB}$ addition $\left(0.828 \mathrm{~d}^{-1}\right)$ and control $\left(0.834 \mathrm{~d}^{-1}\right)$. In contrast, the treatment with the highest addition of GPB shows the lowest growth rate, even though it achieved the highest peak population. This lowest growth rate was significantly different from other treatments. The control, which had the lowest peak population, even had a higher growth rate than this treatment. This phenomenon could be explained in the following paragraph.

As has been discussed before, there was no significant different on the microalgae population among all treatment when it reached their peak point. Even though the control was not spiked with GPB inoculum, the sludge liquor was not sterilized before it was being used. It makes some bacteria; including GP could exist in the medium. Figure 4 indicates that GPB existed in the medium, and it has supported the microalgae productivity. It is the reason why there the peak population of all treatment did not differ significantly. The lowest rate of treatment with $9 \mathrm{ml}$ GPB addition probably because microalgae had to compete with the GPB in uptaking the nutrient. The GPB could enhance the cell productivity by giving growth hormone for the microalgae, while in the same time it compete with the microalgae in grabbing the feed. An ideal treatment according to cell productivity could be fall to $6 \mathrm{ml} \mathrm{GPB}$ addition when it achieved the same population as that of 9 $\mathrm{ml} \mathrm{GPB}$, but had highest growth rate.

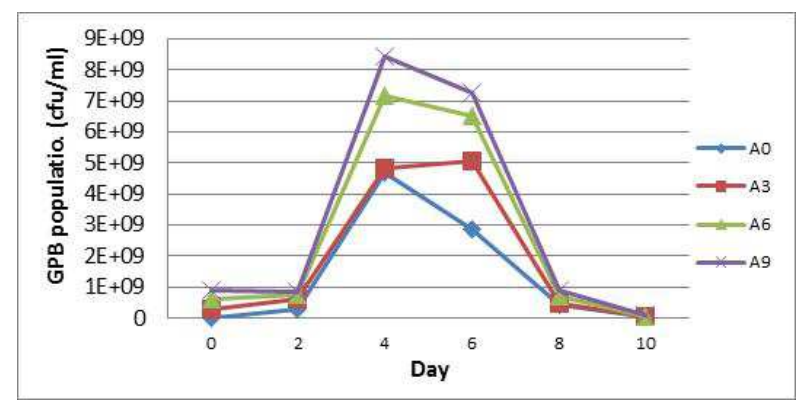

Fig. 4 Population of Azospirillum sp

\section{Lipid Productivity}

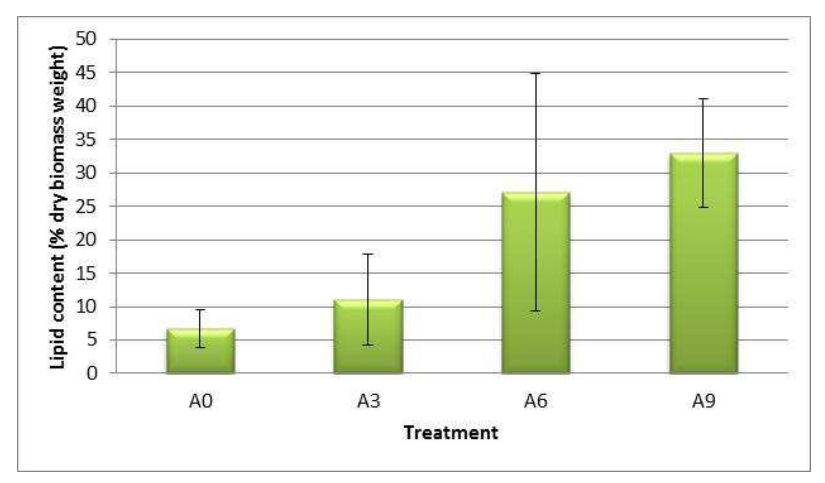

Fig. 5 Lipid content of the microalgae

Fig. 5 shows that the GPB has significantly affect the lipid content of the microalgae during cultivation. It shows that the higher the addition of GPB to the medium, the higher the lipid productivity of the microalgae. The highest lipid value was generated by the treatment of $9 \mathrm{ml} \mathrm{GPB}$ addition with $32.92 \%$ of lipid per dry biomass weight, while the lowest 
was generated by the control $(6.76 \%)$. The $3 \mathrm{ml}$ and $6 \mathrm{ml}$ GPB addition was $27.13 \%$ and $11.03 \%$ of its dry weight, respectively. According to Chisti [7] lipid content microalgae species Chlorella sp. is approximately $28-32 \%$ of the dry weight. Some researchers stated that the lipid productivity of microalgae during the treatment is affected by the nitrogen availability in the medium [8, 9]. They research found that nitrogen starvation could increase the lipid productivity per dry weight of the biomass. Even though chlorella can consume nitrate, ammonium is the dominant nitrogen form that is consumed by the microalgae. By exploring Figure 7, the ammonium content of treatment with $9 \mathrm{ml}$ GPB addition was relatively low during sixth early day of culture. If ammonium amount is divided by the number of existed microalgae, it seems that the ammonium availability of this treatment was the lowest among all treatments. It is probably the answer why the lipid productivity of $9 \mathrm{ml} \mathrm{GPB}$ addition was the highest among all treatments.

\section{Nutrient Concentration of the Medium}

The ten day of ammonium concentration in the medium is shown in figure 7 . The concentration of ammonium decreased in the early culture and increase lately. Ammonium used as nutrients for the growth of microalgae which primarily assist in protein synthesis. In the day-2 to day-4, the ammonium increased insignificantly, and then decreased again in the following day. However, it increased significantly after day-8. The increase of the ammonium after day- 8 is probably because the microalgae as well as the GPB underwent a death phase. When those two microorganisms dead, decomposition could occur in the medium by the decomposer bacteria that might has been existed in the medium, due to unsterile liquor. This reason could also be applied to the nitrate concentration that also decrease in the first 8 days then increased later (Fig. 8). Even though ammonium and nitrate is needed by microalgae, too high microalgae concentration is toxic to microalgae. Each type of microalgae has different ability to survive in the different level of ammonium and nitrate.

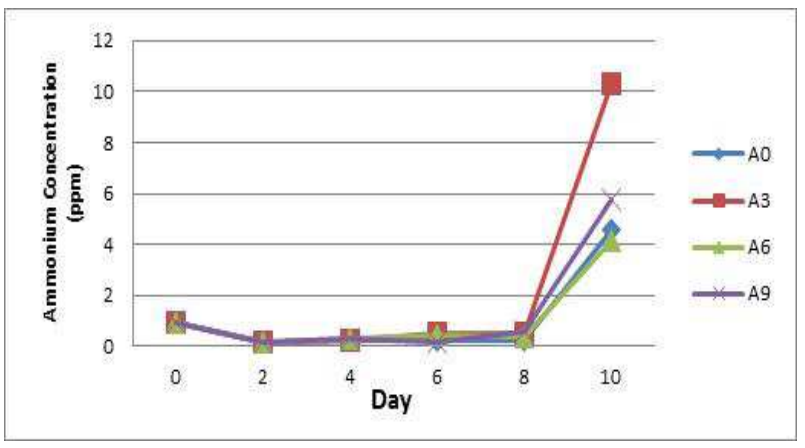

Fig.6. Ammonium concentration in the medium

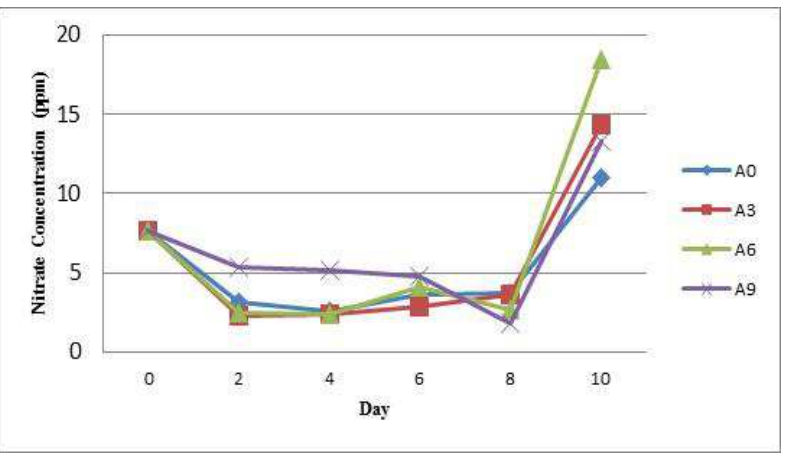

Fig. 7. Nitrate Concentration in the medium

Orthophosphate is one of the phosphate forms in the water. Orthophosphate elements are needed to improve the productivity of phytoplankton. Figure 9 shows that the orthophosphate content in the medium was between 0.57 to $1.92 \mathrm{ppm}$. The concentration of phosphate decreased on the first two day of culture. After experiencing a decline, the content of orthophosphate increased again the next day. Smallest decline occurred in the control. When the content of phosphate in the waters exceed the normal requirements of microorganisms, it could stimulate excessive growth of other organism that leads to anaerobic condition. It usually follows by the dead of aerobic microorganism.

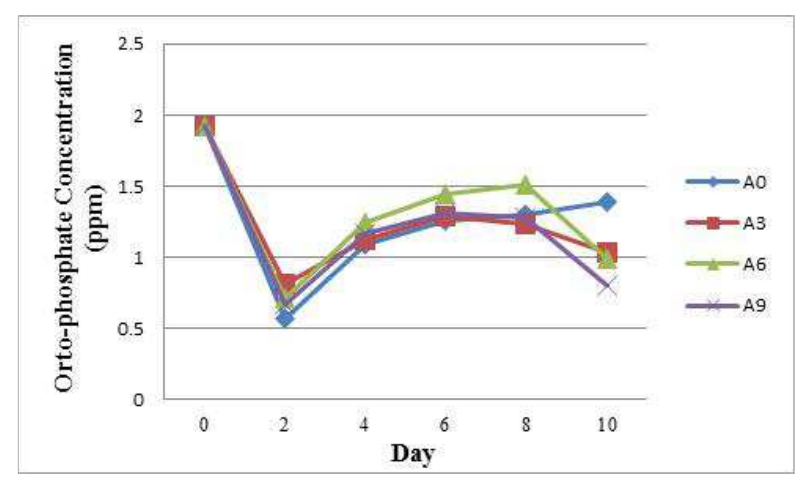

Fig. 8. Ortho-phosphate concentration in the medium

\section{CONCLUSIONS AND SUGGESTION}

According to the research, the addition of GPB has affected the peak population of microalgae, but was not statistically significant. The highest growth rate achieved by $6 \mathrm{ml}$ GPB addition, while too many GPB even decreased the growth rate due to the competition to uptake nutrient. Lipid productivity was affected significantly by the number of GPB added to the medium. However, it seems that GPB was affect indirectly, i.e. by exhausting the nitrogen that leads to the higher lipid productivity. There was no explanation whether secreted hormone from GPB has influenced this lipid productivity.

To ensure that nutrient from the sludge is the only nutrient that is consumed by microalgae, sterilization of sludge prior to applying to the culture is necessary to be done. 


\section{REFERENCES}

[1] M. M. Teresa, A. M. Antonio, and N.S. Caetano. Microalgae for Biodiesel Production and Other Applications: A Review. Renewable and Sustainable Energy, 14 217-232, 2010.

[2] E.S. Umdu, M. Tuncer, E. Seker. Transesterification of Nannochloropsis oculata microalga's lipid to biodiesel on Al2O3 supported $\mathrm{CaO}$ and $\mathrm{MgO}$ catalysts. Bioresource Technology Volume 100, Issue 11, June 2009, Pages 2828-2831. 2009.

[3] A. E. Abdelaziz, G. B. Leite, M. A. Belhaj and P. C. Hallenbeck. Screening microalgae native to Quebec for wastewater treatment and biodiesel production. Bioresource Technology, 157, 140-148. 2014

[4] I., Woertz, A.. Feffer, T. Lundquist and Y. Nelson. Algae Grown on Dairy and Municipal Wastewater for Simultaneous Nutrient Removal and Lipid Production for Biofuel Feedstock. Journal of Environmental Engineering, 135, 1115-1122. 2009

[5] L. E. De-Bashan, H. Antoun and Y. Bashan. Involvement of indole-3acetic acid produced by the growth-promoting bacterium azospirillum spp. In promoting growth of chlorella vulgaris. Journal of Phycology, 44, 938-947. 2008

[6] L. E. De-Bashan, J.P. hernandez, T. Morey and Y. Bashan Microalgae growth-promoting bacteria as a helper for microalgae: a novel approach for removing ammonium and phosphorus from municipal wastewater. Water Research, 38, 466-474. 2004

[7] Y. Chisti. Biodiesel from microalgae. Biotechnology Advances, 25 294-306. 2007

[8] S. Ho, C. Chen, J. Chang. Effect of light intensity and nitrogen starvation on $\mathrm{CO} 2$ fixation and lipid/carbohydrate production of an indigenous microalga Scenedesmus obliquus CNW-N. Bioresource Technology, Volume 113, June 2012, Pages 244-252. 2012.

[9] Y. Li, M. Horsman, B. Wang, N. Wu, C. Q. Lan. Effects of nitrogen sources on cell growth and lipid accumulation of green alga Neochloris oleoabundans. Microbiology and Biotechnology, December 2008, Volume 81, Issue 4, pp 629-636. 2008. 